\title{
Virtual hybrid human body model for PTW safety assessment
}

\author{
L. Hynčík ${ }^{a, *}$, T. Bońkowski ${ }^{a}$, J. Vychytil ${ }^{b}$

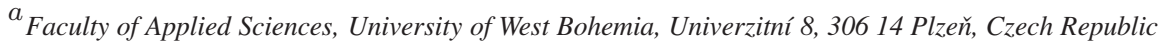 \\ ${ }^{b}$ New Technologies for the Information Society, Faculty of Applied Sciences, University of West Bohemia, \\ Technická 8, 30614 Plzeň, Czech Republic \\ Received 28 July 2017; accepted 2 December 2017
}

\begin{abstract}
Road traffic accidents cause one of the highest numbers of severe injuries. The numbers of deaths and seriously injured citizens prove that traffic accidents and their consequences are still a serious problem to be solved. Virtual human body models play an important role to assess injuries during impact loading especially for scenarios, where complex dynamical loading is taken into account. The most suffering group is so called vulnerable road users (VRU) like powered two-wheelers (PTW) riders. The presented work contributes to increasing safety of PTW riders by implementing virtual human body model for injury risk analysis. The scalable hybrid virtual human body model Virthuman, which was formerly developed, validated and demonstrated in impact scenarios, is improved by updated neck and shoulder models in order to describe the realistic kinematics during complex long duration impact loading and presented in the oblique impact scenario compared to the THUMS results.
\end{abstract}

(c) 2017 University of West Bohemia. All rights reserved.

Keywords: virtual human body model, PTW, neck, shoulder, oblique impact

\section{Introduction}

WHO [17] monitors that every year the lives of approximately 1.25 million people are cut short as a result of a road traffic crash. Between 20 and 50 million more people suffer non-fatal injuries, with many incurring a disability as a result of their injury. Nearly half of those dying on the world's roads are so called vulnerable road users (VRU): pedestrians, cyclists, and motorcyclists including powered two-wheeler (PTW) riders.

Road traffic injuries cause considerable economic losses to individuals, their families, and to nations as a whole [17]. These losses arise from the cost of treatment as well as lost productivity for those killed or disabled by their injuries, and for family members, who need to take time off work or school to care for the injured. Road traffic crashes cost most countries $3 \%$ of their gross domestic product [17].

VRU including PTW riders usually undergo complex kinematics during the impact loading, because they are usually not protected by active safety systems preventing collisions and passive safety elements like seat belts and airbags. Existing human body models [18] are usually developed to cover standard impact scenarios as car frontal and side impacts. Whilst basic multi-body (MB) models can serve for fast calculation of global kinematics without any detailed description of the scenario, finite element (FE) models consume a lot of computational time. Therefore hybrid approach is a useful compromise.

*Corresponding author. Tel.: +420 377634 709, e-mail: hyncik@ntc.zcu.cz. https://doi.org/10.24132/acm.2017.389 
Virthuman $[7,15]$ is a fully validated scalable virtual hybrid biomechanical human body model coupling the basic MB structure (MBS) with deformable segments, resulting in short calculation time, hence it is suitable for calculating longer impact scenarios, which VRU generally are.

\section{Method}

\subsection{Basic model}

The current model Virthuman [7,15], see Fig. 1, concerns the skeleton of the whole model created as MBS and the skin surface segmented into the rigid surface parts (so called superelements) interconnected by strips of elements without any mechanical response. The superelements are fixed to the basic MBS structure by springs and dampers tuned to assure biofidelic deformation for particular impacts. The main advantage of the MBS method is reduced computational time.

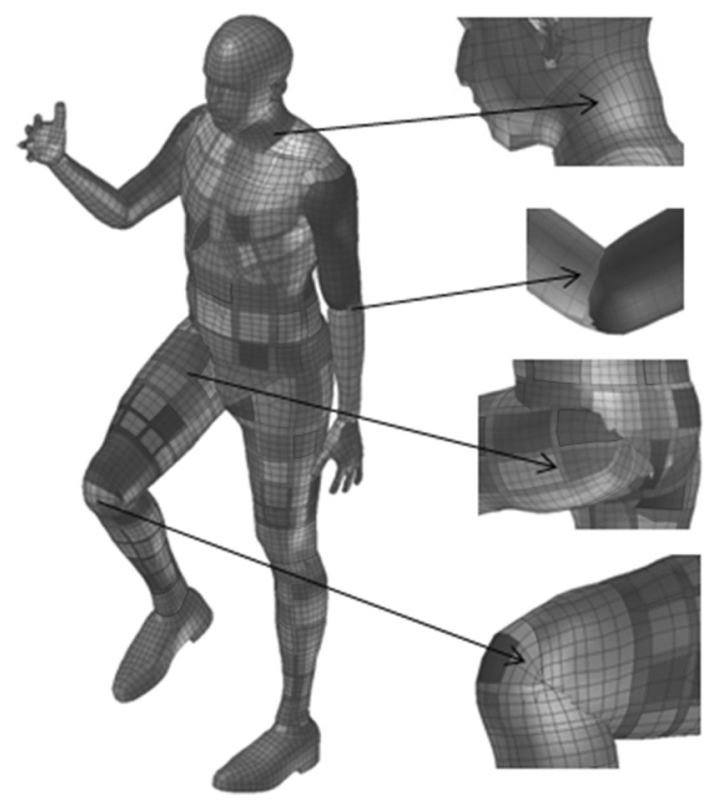

Fig. 1. Virthuman [15]

The Virthuman model is validated [15] and used for prediction of injury risk in various not only road traffic scenarios $[9,10,16]$. For the PTW impact scenarios, the head, neck and extremities usually undergo complex multi-directional loading due to the fact that the body moves almost freely by inertia in the space. The presented work further develops the model [14] suitable for pedestrian testing by updating the neck and shoulder for PTW riders.

\subsection{Neck model}

The Virthuman neck model is separated to 7 vertebrae (see Fig. 2) interconnected by 6 joints. Another two joints are necessary to connect the neck with the head and the torso, each of them having 6 degrees of freedom defined by nonlinear stiffness and damping. To assure realistic biofidelic kinematics of the head-neck complex, the range of motion (ROM) of particular joints were set according to the physiological data [4]. Since scenarios with VRU, such as pedestrian, vehicle or PTW impacts, exhibit excessive neck deformations, the stiffness characteristics of individual joints close to the ROM values are modified [4] without violation of validations for $15 \mathrm{~g}$ frontal and $7 \mathrm{~g}$ lateral sled tests. Fig. 2 right shows an example for axial rotation of the $\mathrm{C} 3 / \mathrm{C} 4$ join updated by the particular ROM for this joint. 

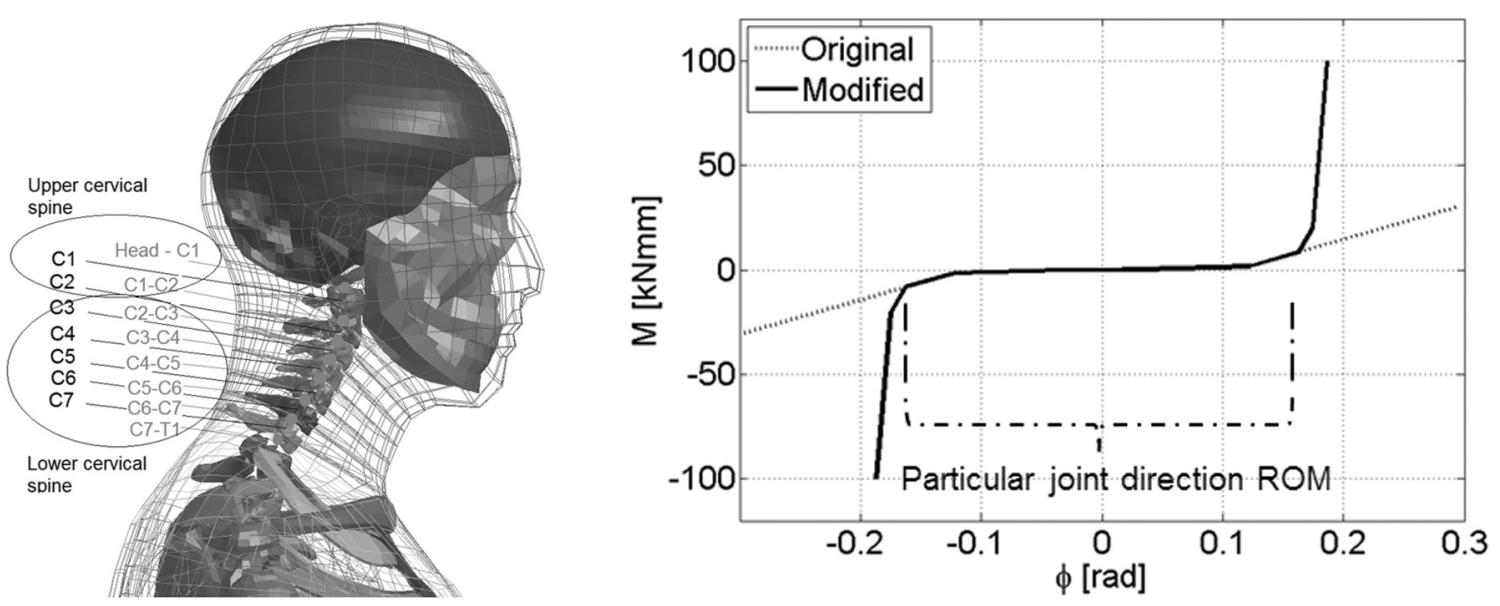

Fig. 2. Improved neck (left: model, right: updated ROM for axial C3/C4 rotation) $[11,13]$

The validation is based on the sled test environment $[4,12]$, where the head and the neck are separated from the model including all the neck vertebrae as well as the first thoracic vertebra $\mathrm{T} 1$ [15] that is loaded by $15 \mathrm{~g}$ frontal and $7 \mathrm{~g}$ lateral pulse. The head spatial displacement is monitored. Further, the performance of the neck model in the rigid wall $50 \mathrm{~km} / \mathrm{h}$ impact is analysed.

\subsection{Shoulder model}

For standard sled test environment, where upper extremities do not play a considerable role, the shoulder complex can be simplified by a single joint with well validated ROM. However, for VRUs' accident scenarios, where the human body suffers from complex motion, the proper shoulder response is necessary.

In order to describe the complex shoulder motion due to the upper arm impact, the upper arm was composed by 2 rigid bodies (see Fig. 3). The first one creates the skeleton of the whole MBS structure, whilst the second one is linked to the first one enabling the compressibility of the upper arm complex and proper behaviour of the shoulder under impact loading. The compression parameters were tuned in order to fit the experimental corridor.
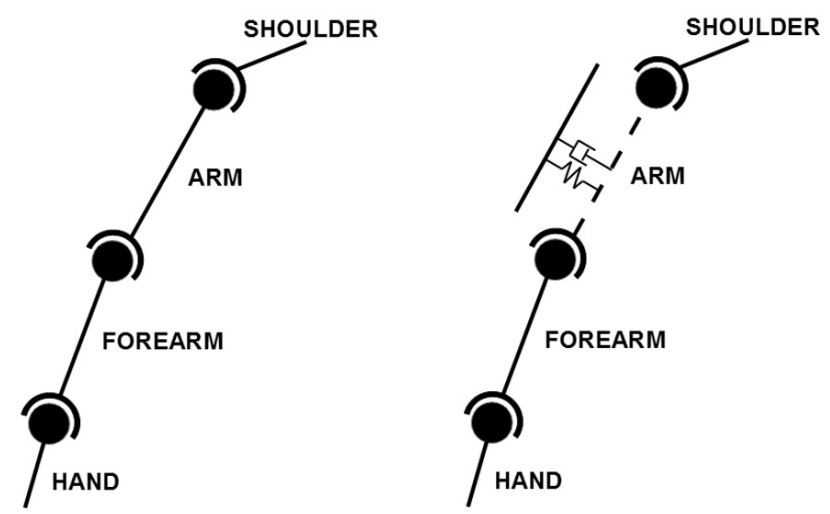

Fig. 3. Upper arm model (left: original, right: improved) 

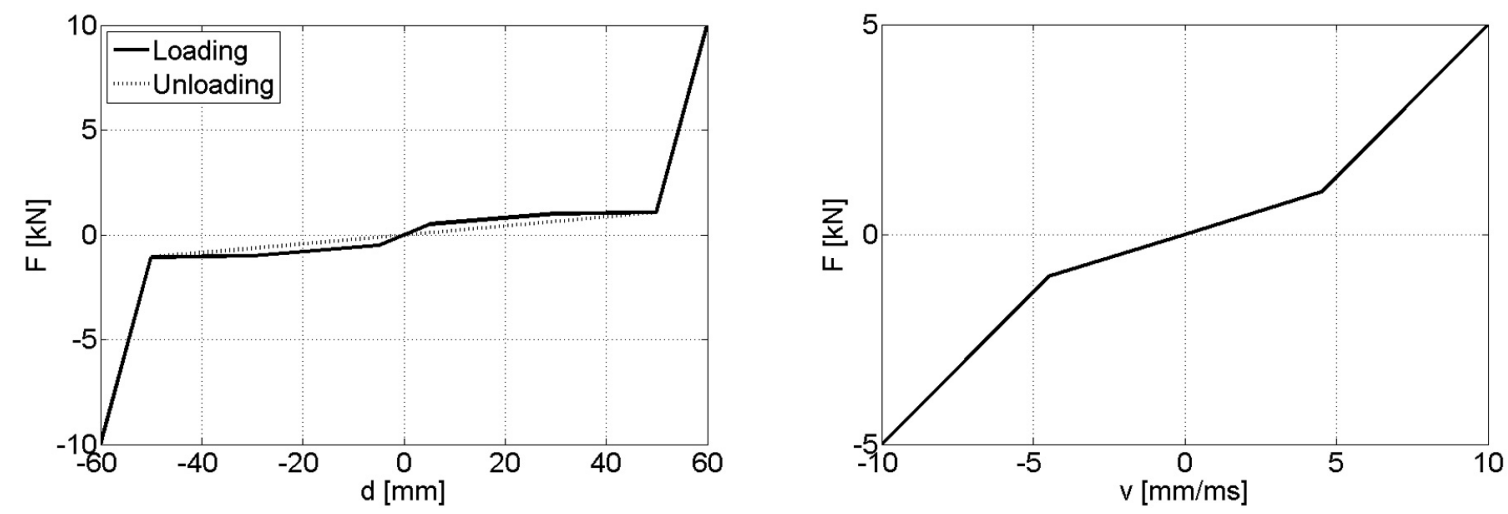

Fig. 4. Shoulder joint loading and unloading (left) and damping (right)

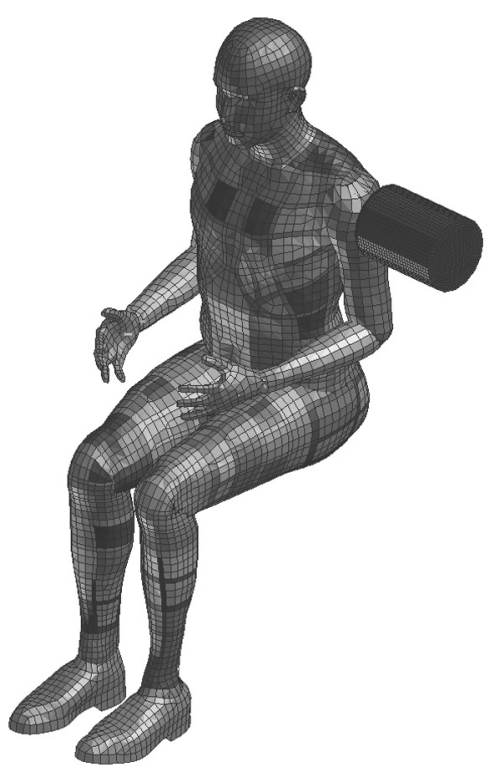

Fig. 5. Shoulder validation setup [1]

The shoulder is validated according to [1]. The model is seated upright with no additional lateral supports on a flat horizontal rigid surface with the legs straight and parallel. The arms are positioned parallel to the thorax. The cylindrical impactor of the mass $23.4 \mathrm{~kg}$ and the diameter of $150 \mathrm{~mm}$ hits the shoulder laterally with the impact velocity of $4.5 \mathrm{~m} / \mathrm{s}$. The axis of the impactor is aligned with the shoulder pivot and at $90^{\circ}$ to the mid sagittal plane, see Fig. 5. The contact force and the maximum deflection are monitored.

\subsection{Impact testing}

In most motorcycle accidents, the body angle is $30^{\circ}$ or less towards the ground [3]. Therefore the body is tested in oblique impact according to [6], where the analysis with the full validated FE model THUMS [11] is provided for comparison to the Virthuman hybrid model. The model is loaded by gravity and varying impact normal $v_{n}$ and tangential $v_{t}$ (side) velocity. The test setup is shown in Fig. 6. 


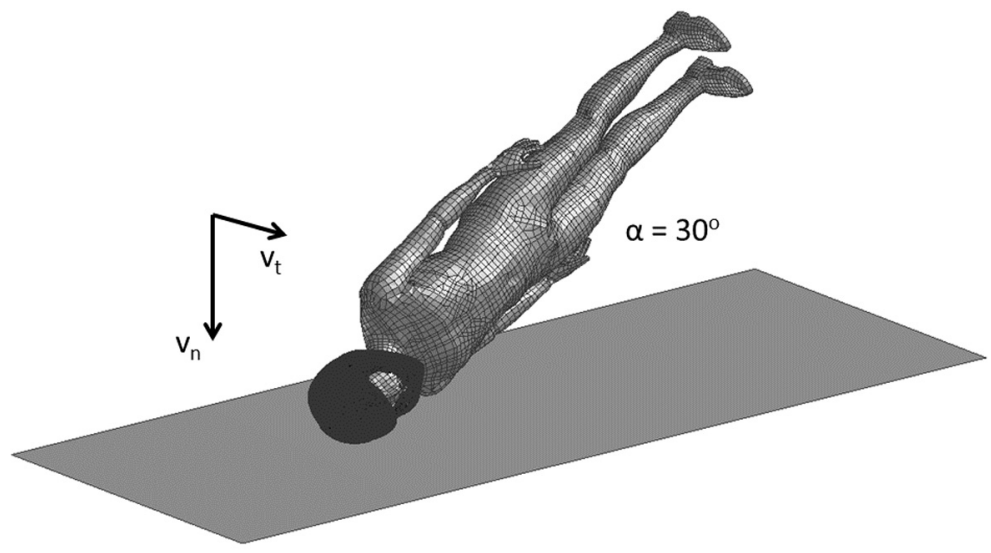

Fig. 6. Oblique impact test setup

The Virthuman model is scaled to the THUMS anthropometry $(175 \mathrm{~cm}, 77 \mathrm{~kg}$ [8]). Since the scaling algorithm desires age [7], the age group chosen is 30-35 years, because there the average height (percentile 50) equals to $176 \mathrm{~cm}$ and average mass equals to $78 \mathrm{~kg}$, which are the closest values for scaling.

The medium size FE helmet model developed within the MYMOSA project $[5,6]$ is coupled to the human head. Due to the head and helmet geometry, minor mesh corrections on the head are done to avoid penetrations to position the helmet with a small constant gap between the helmet liner and the head. This gap is usually filled by the foam in the reality, however, negligible effect of the missing foam is shown [6]. The helmet chin strap is passed under the chin and tightened by the pretension force of $5 \mathrm{~N} \mathrm{[6]} \mathrm{and} \mathrm{there} \mathrm{is} \mathrm{defined} \mathrm{a} \mathrm{sliding} \mathrm{contact} \mathrm{between} \mathrm{the} \mathrm{liner} \mathrm{and}$ the head with friction coefficient equal to 0.2 [6].

\section{Results}

\subsection{Neck results}

The model with updated neck joints ROM shows the proper biofidelic performance. Whilst the kinematic performance of the improved neck model in the frontal rigid wall $50 \mathrm{~km} / \mathrm{h}$ impact is shown in Fig. 7 its responses in the both $7 \mathrm{~g}$ and $15 \mathrm{~g}$ impacts [15] are not violated as shown in Fig. 8 and Fig. 9.

During the unloading phase after $20 \mathrm{~ms}$, the response goes outside the corridors, which is probably caused by the model simplicity. However, the injuries are related for the maximum values during the loading, so the response at the end shall not violate the injury prediction.
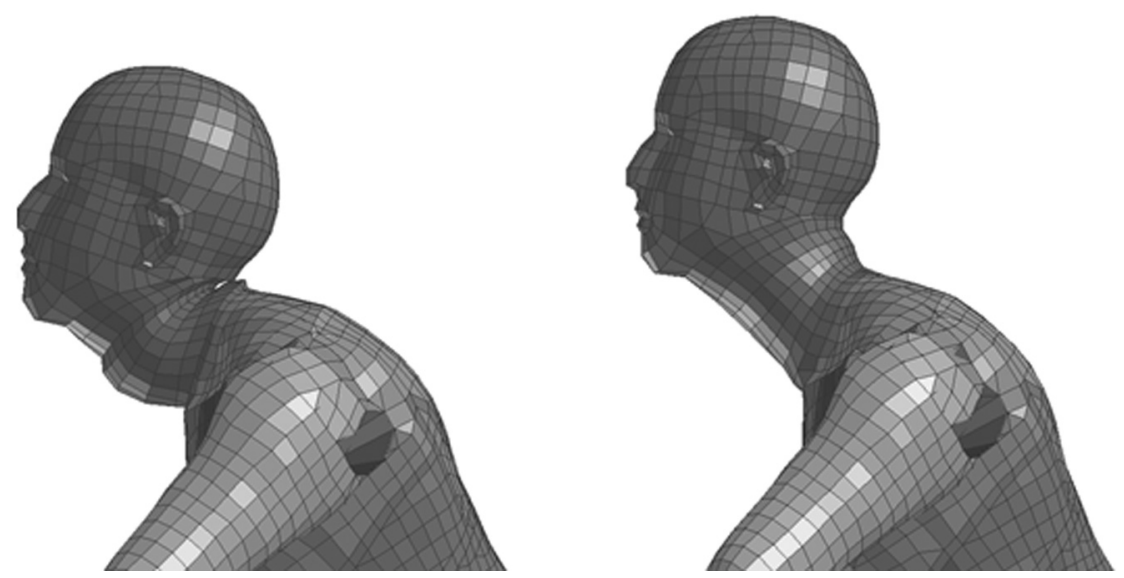

Fig. 7. Neck behaviour (left: original neck, right: improved neck) 

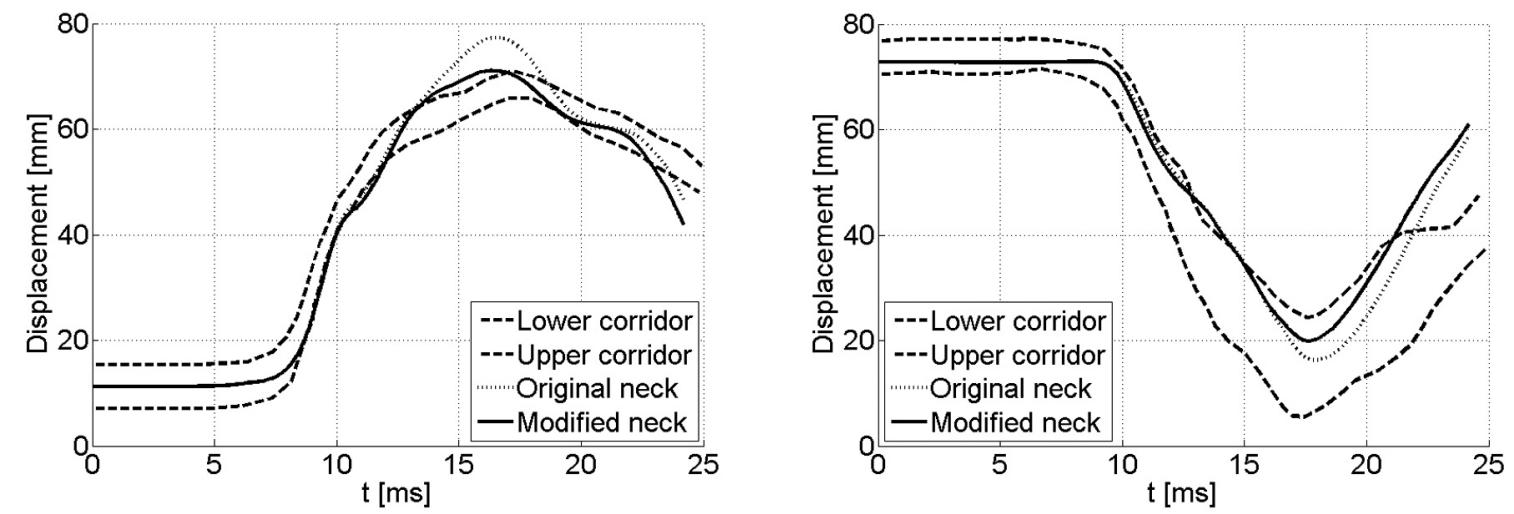

Fig. 8. Head displacement at $15 \mathrm{~g}$ frontal impact test (left: frontal direction, right: vertical direction)
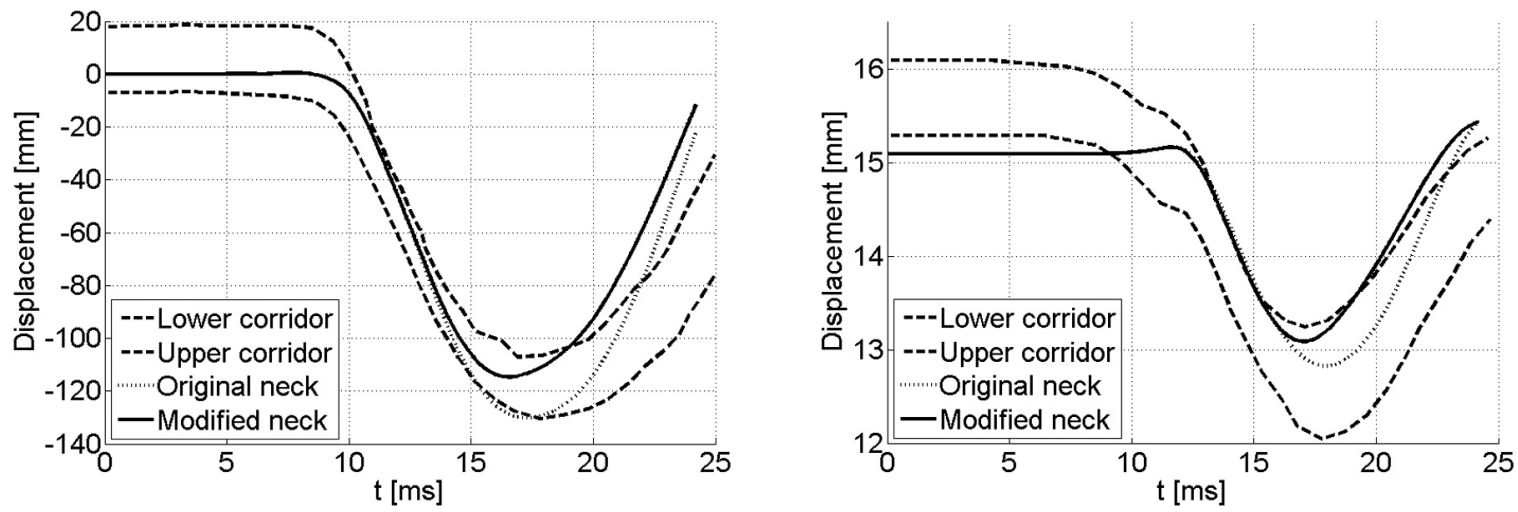

Fig. 9. Head displacement at $7 \mathrm{~g}$ lateral impact test (left: lateral direction, right: vertical direction)

\subsection{Shoulder results}

The time dependent contact force is shown in Fig. 10. In general, the predicted curve is within the corridor showing a good agreement with experimental data.

At the end of the impact, the force drops slightly below the corridor. This may be caused by shrinking the contact area between the impactor and the shoulder, i.e. the contact area in the model (rigid impactor on the rigid segment) is smaller than the contact area in the real experiment (soft tissues of the shoulder adjusting to the flat surface of the rigid impactor). The maximum displacement should be within the range of $37.5 \mathrm{~mm} \pm 3.5 \mathrm{~mm}$ [2]. The Virthuman model obtains the value of $41.9 \mathrm{~mm}$ which is appropriate.

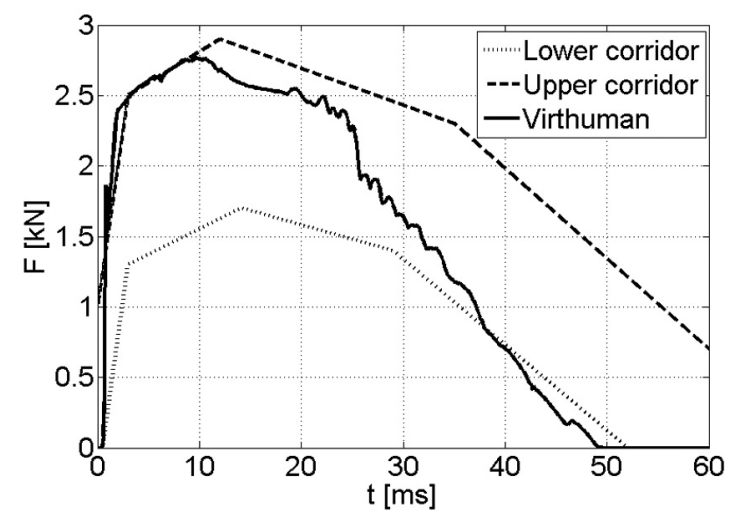

Fig. 10. Shoulder contact force 


\subsection{Impact testing}

Combination of normal and tangential velocities brings complex kinematics of the head and neck after the ground impact. The performance of the scaled virtual human body Virthuman (VH) in the oblique impact is compared to the THUMS model by means of maximum tangential and normal contact force $\left(F_{t, \text { max }}\right.$ and $F_{n, \text { max }}$, respectively), their ratio $F_{t, \text { max }} / F_{n, \text { max }}$ and maximum head acceleration $a_{\max }[6]$, see Table 1 .

The simulations show comparable results for all the normal and tangential velocities. For the increasing normal velocity, the effect of the MBS approach used for developing the Virthuman model is considerable in stiffer response and higher contact forces. However, for evaluation of head acceleration and the potential injury assessment, the values of maximum head acceleration are fully in line with those performed using the FE model THUMS.

Table 1. Comparison between VH and THUMS [6]

\begin{tabular}{|c|c|c|c|c|c|c|c|c|c|}
\hline \multicolumn{7}{|c|}{ Impact velocity } & \multicolumn{7}{|c|}{ Contact force } & \multicolumn{2}{c|}{ Head acceleration } \\
\hline \multirow{2}{*}{$v_{n}[\mathrm{~m} / \mathrm{s}]$} & \multirow{2}{*}{$v_{t}[\mathrm{~m} / \mathrm{s}]$} & $F_{n, \max }[\mathrm{kN}]$ & \multicolumn{2}{|c|}{$F_{t, \max }[\mathrm{kN}]$} & $F_{t, \max } / F_{n, \max }[-]$ & \multicolumn{2}{|c|}{$a_{\max }[\mathrm{g}]$} \\
\cline { 3 - 10 } & & $\mathrm{VH}$ & THUMS & VH & THUMS & VH & THUMS & VH & THUMS \\
\hline \multirow{2}{*}{2.5} & 5 & 4.27 & 3.82 & 1.59 & 1.31 & 0.37 & 0.33 & 67 & 73 \\
\cline { 2 - 11 } & 10 & 4.40 & 3.85 & 1.75 & 1.54 & 0.40 & 0.40 & 68 & 73 \\
\hline \multirow{2}{*}{5} & 5 & 9.93 & 7.90 & 3.01 & 2.21 & 0.30 & 0.22 & 132 & 144 \\
\cline { 2 - 10 } & 10 & 9.97 & 7.68 & 3.96 & 3.08 & 0.40 & 0.38 & 136 & 143 \\
\hline \multirow{2}{*}{7.5} & 5 & 12.88 & 11.54 & 4.09 & 3.32 & 0.32 & 0.18 & 190 & 199 \\
\cline { 2 - 10 } & 10 & 12.52 & 11.72 & 4.72 & 3.50 & 0.38 & 0.31 & 190 & 191 \\
\hline
\end{tabular}

\section{Conclusion}

The work shows the improvement of the scalable human body model Virthuman by adopting compressible shoulder and improving ROM in the neck skeleton in order to sustain the validation of the VRU scenario impact.

The performed validation for both above mentioned segments shows not only appropriate response of the Virthuman model, but also better kinematics for the head/neck complex, which is one of the most injury suffering part for VRU.

The advantage of the Virthuman model is its low time consumption due to the long duration calculations. The full body validation in oblique impact for the wide spectra of normal and tangential velocities proves that the Virthuman model is suitable for analysing complex impact scenarios.

\section{Acknowledgements}

The work has been supported by the research project LTC17001 "Exploitation of virtual human model for reducing injury risk of PTW riders" as the national link to the COST Action TU1407 "Scientific and technical innovations for safer Powered Two Wheelers (PTW)" and the internal grant project SGS-2016-059 "Computer modelling and monitoring of human body used for medicine". 


\section{References}

[1] Bendjellal, F., Walfisch, G., Fayon, A., Tarriere, C., APR biomechanical data, APR, Nantere, France, 1984.

[2] Bours, R.C.H., Campen, V.D., Wismans, J.S.H.M., Bovendeerd, P.H. M., Happee, R., Veeger, H.E.J., Mathematical human body modeling in impact: Shoulder and upper extremity, Eindhoven University of Technology, Master thesis, 2000.

[3] COST327 - Motorcycle safety helmets, Final Report of the Action, Belgium, 2001.

[4] De Jager, M. K. J., Mathematical head-neck models for acceleration impacts, Eindhoven University of Technology, Ph.D. thesis, 1996.

[5] Ghajari, M., Galvanetto, U., Iannucci, L., Wilinger, R., Influence of the body on the response of the helmeted head during impact, International Journal of Crashworthiness 16 (3) (2011) 285-295. https://doi.org/10.1080/13588265.2011.559798

[6] Ghajari, M., Peldschus, S., Galvanetto, U., Iannuccia, L., Effects of the presence of the body in helmet oblique impacts, Accident Analysis and Prevention 50 (2013) 263-271. https://doi.org/10.1016/j.aap.2012.04.016

[7] Hynčík, L., Čechová, H., Kovář, L., Bláha, P., On scaling virtual human models, SAE Technical Paper 2013-01-0074, 2013. https://doi.org/10.4271/2013-01-0074

[8] Iwamoto, M., Nakahira, Y., Tamura, A., Kimpara, H., Watanabe, I., Miki, K., Development of advanced human models in THUMS, Proceedings of the 6th European LS-DYNA Users' Conference, Gothenburg, 2007, pp. 47-56.

[9] Lindstedt, L., Vychytil, J., Dziewonski, T., Hynčík, L., Numerical tests of the virtual human model response under dynamic load conditions defined in federal aviation regulation: Part 23.562 and 25.562 - Preliminary Study, Archive of Mechanical Engineering 63 (4) (2016) 511-530. https://doi.org/10.1515/meceng-2016-0029

[10] Maňas, J., Kováŕ, L., Petřík, J., Čechová, H., Špirk, S., Validation of human body model VIRHUMAN and its implementation in crash scenarios, Advances in Mechanisms Design - Proceedings of TMM 2012, Springer, 2012, pp. 351-356.

[11] THUMS, Am50 pedestrian model: Version 3, 0-080225, Toyota Central R\&D Labs, 2008.

[12] Van der Horst, M. J., Human head neck response in frontal, lateral and rear end impact - modelling and validation, Eindhoven University of Technology, Ph.D. thesis, 2002.

[13] Vychytil, J., Čechová, H., Maňas, J., Mechanical response of neck in virtual human body model VIRTHUMAN, Proceedings of the 31st Conference with International Participation Computational Mechanics, Pilsen, University of West Bohemia, 2015, pp. 129-130.

[14] Vychytil, J., Hynčík, L., Maňas, J., Pavlata, P., Striegler, R., Moser, T., Valášek, R., Prediction of injury risk in pedestrian accidents using virtual human model VIRTHUMAN: Real case and parametric study, SAE Technical Paper 2016-01-1511, 2016. https://doi.org/10.4271/2016-01-1511

[15] Vychytil, J., Maňas, J., Čechová, H., Špirk, S., Hynčík, L., Kovář, L., Scalable multi-purpose virtual human model for future safety assessment, SAE Technical Paper 2014-01-0534, 2014. https://doi.org/10.4271/2014-01-0534

[16] Vychytil, J., Špička, J., Hynčík, L., Maňas, J., Pavlata, P., Striegler, R., Moser, T., Valášek, R., Novel approach in vehicle front-end modeling for numerical analyses of pedestrian impact scenarios, SAE Technical Paper 2017-01-1451, 2017. https://doi.org/10.4271/2017-01-1451

[17] WHO, Road traffic injuries, May 2017, http://www.who.int/mediacentre/factsheets/fs358/en.

[18] Yang, K. H., Hu, J., White, N. A., King, A. I., Chou, C. C., Prasad, P., Development of numerical models for injury biomechanics research: A review of 50 years of publications in the stapp car crash conference, Stapp Car Crash Journal 50 (2006) 429-490. 